\title{
Bacillus cereus cellulitis from contaminated heroin
}

\author{
S. J. DANCER, D. MCNAIR, P. FINN* and A-B. KOLSTO† \\ Departments of Microbiology and Surgery*, Vale of Leven Hospital, Alexandria, Dunbartonshire, G83 OUA and \\ $\uparrow$ Biotechnology Centre of Oslo and Institute of Pharmacy, University of Oslo, 1125 Blindern, 0316 Oslo, \\ Norway
}

\begin{abstract}
Concern exists over recent unexplained deaths among intravenous drug users. This report describes a patient with crepitant cellulitis who was admitted complaining of severe pain in the right forearm. Ultrasonography demonstrated gas in the tissues and he was referred for early surgical debridement of the arm. He was treated with intravenous benzyl penicillin, gentamicin and metronidazole and made a full recovery. Aspirate samples grew Bacillus cereus, morphologically similar to the isolate obtained from a sample of the patient's own heroin. Antibiogram and API 50CHB profiles were also similar. Further typing included ' $H$ ' flagellar serotyping, which found both blood and heroin strains to be non-typable, and amplified fragment polymorphism analysis, which showed that the strains were indistinguishable. Genotyping of two selected genes from $B$. cereus confirmed almost certain identity between the two strains. This case illustrates the potential virulence of $B$. cereus when inoculated into tissues, and to our knowledge, is the first report to demonstrate a conclusive microbiological link between contaminated heroin and serious sepsis in a drug user due to $B$. cereus.
\end{abstract}

\section{Introduction}

Skin 'popping' is well known among chronic drug abusers [1]. It occurs when drugs are injected straight into the tissues rather than into a blood vessel, either by accident or as a result of diminished vascular access in long-term addicts. This can have serious effects if the material is microbiologically contaminated, as bacteria introduced directly into the tissues are provided with an ideal culture medium. Dissolving drugs in commercially available acids, such as citric or tartaric, serves to enhance the breakdown of tissues at the injection site and thus may accelerate bacterial growth. Drug users are already at risk of infection from dirty injecting paraphernalia and equipment sharing [2].

Bacillus cereus has already been implicated in serious infections in drug users [3]. It has been isolated from clinical specimens, injecting paraphernalia and heroin samples [3, 4]. The organism is known to cause a gas gangrene-like syndrome and has been confused with Clostridium spp., the more usual aetiology associated with gas gangrene [5-8]. Both organisms appear as gram-positive rods on microscopy, and both produce

Received 17 April 2001; revised version accepted 30 Sept. 2001.

Corresponding author: Dr S.J. Dancer (e-mail: Stephanie.dancer@vol.scot.nhs.uk). several toxins known to exert clinical effects. Both are found in soil and form spores able to survive the harshest of environmental conditions. Thus, they are likely contaminants in heroin and associated injecting equipment $[4,9]$. Furthermore, the dissolution of street heroin in an acid medium before injection destroys vegetative bacteria whilst permitting survival of spores.

The following case report describes a case of serious sepsis in a drug user, caused by $B$. cereus originating from his own supply of heroin.

\section{Case report}

A 28-year-old chronic heroin user with hepatitis C presented with general malaise, vomiting and a painful swollen right forearm. He had many prior admissions due to injection site sepsis and was using $3-5 \mathrm{~g}$ of heroin weekly, which he dissolved in citric acid. Two days before admission, he injected heroin into subcutaneous fat and muscle below the right antecubital fossa. This batch of heroin had already been used previously with no adverse effects. On admission, general inspection showed him to be pale, sweaty and dyspnoeic. He was apyrexial $\left(36.1^{\circ} \mathrm{C}\right)$, with a blood pressure of $95 / 55 \mathrm{mmHg}$ and pulse rate of 88 beats/ min. He appeared dehydrated and was tremulous. There was a palpable tender liver edge in the right 
hypochondrium and the white cell count was $22 \times$ $10^{9} / \mathrm{L}$ with a neutrophilia of $20.8 \times 10^{9} / \mathrm{L}$. Liver function tests taken on admission were abnormal: aspartate transaminase (SGOT) $248 \mathrm{IU} / \mathrm{L}$ (normal range 5-40 IU/L), alanine transaminase (SGPT) 254 IU/L (2-40 IU/L), alkaline phosphatase $641 \mathrm{IU} / \mathrm{L}$ (60-270 IU/L) and $\gamma$-glutaryl transaminase (GGTP) 357 IU/L (4-45 IU/L).

His right forearm was erythematous, indurated and tender to palpation over an area of $6.0 \times 8.0 \mathrm{~cm}$. There was no evidence of skin necrosis, nor palpable evidence of fluctuance or crepitus. Other injection sites were clinically unremarkable. In view of recent reports of drug-related deaths from sepsis [10], he underwent an urgent ultrasound examination, which demonstrated thickening of the subcutaneous fat planes in the right forearm (10.0 mm right versus $2.5 \mathrm{~mm}$ left). Within the fat were patchy areas of hypo-echogenicity and several small echogenic foci suggestive of gas. In contrast, the left forearm appeared normal. Blood cultures were taken. He was resuscitated with crystalloids, intravenous antibiotics (benzyl penicillin 2.4 MU, gentamicin $400 \mathrm{mg}$ and metronidazole $500 \mathrm{mg}$ ) and referred for urgent surgical debridement.

Fine needle aspiration of the swollen forearm was attempted before surgery and the aspirated material was inoculated directly into culture media. Full-thickness skin excision was performed under general anaesthesia, debriding piecemeal back to viable tissue on all sides. Underlying muscle and its fascia were not involved. There was thickening and oedema of the subcutaneous fat showing patchy areas of necrotic discolouration. The wound was lavaged with saline and the edges of the defect were loosely approximated with a single absorbable suture.

The following day, his white cell count fell to $12 \times 10^{9} / \mathrm{L}$. The patient provided some of the heroin used before admission, and a small amount of a grey/ brown powder was referred to the microbiology laboratory for analysis. He was discharged well 5 days later and defaulted from follow-up, but subsequently attended the hospital on a further three occasions with injection site infections.

\section{Microbiology - methods and results}

\section{Patient}

Aspirate samples were processed according to laboratory Standard Operating Procedures. Anaerobic cultures signalled positive at $48 \mathrm{~h}$ and initial microscopy revealed gram-positive rods. Aerobic cultures required a further $48 \mathrm{~h}$ before signalling bacterial growth; again, gram-positive rods were seen on microscopy from the aspirate. Tissue obtained at operation and blood cultures failed to produce any bacterial growth.
All cultures were plated on to blood, chocolate and fastidious anaerobe agar and incubated in air with $\mathrm{CO}_{2}$ $5 \%$ for $24 \mathrm{~h}$ and anaerobically for $48 \mathrm{~h}$. Discrete colonies of Bacillus spp. were retrieved and identified as $B$. cereus by selective agar [11] and the API 50 CHB identification system [12]. The Stokes method was used for antibiotic susceptibility testing, with Staphylococcus aureus NCTC 6571 as a control. Disk strengths were penicillin $1 \mathrm{IU}$, erythromycin $5 \mu \mathrm{g}$, amoxicillin $10 \mu \mathrm{g}$, cephradine $30 \mu \mathrm{g}$, gentamicin $10 \mu \mathrm{g}$ and vancomycin $30 \mu \mathrm{g}$. Isolates were stored in cryopreservative at $-70^{\circ} \mathrm{C}$ for further study.

\section{Heroin}

About $100 \mathrm{mg}$ of grey-brown powdered heroin was received in the laboratory; approximately half was placed in Robertson's cooked meat broth and incubated for $48 \mathrm{~h}$ before subculture on to blood agar incubated in $\mathrm{CO}_{2} \quad 5 \%$ and anaerobically. Discrete colonies of Bacillus spp. were visible at $48 \mathrm{~h}$ on the anaerobic plates, and these were confirmed as B. cereus as described previously. The heroin isolate appeared morphologically similar on culture and API profile to the patient isolate and both produced an identical

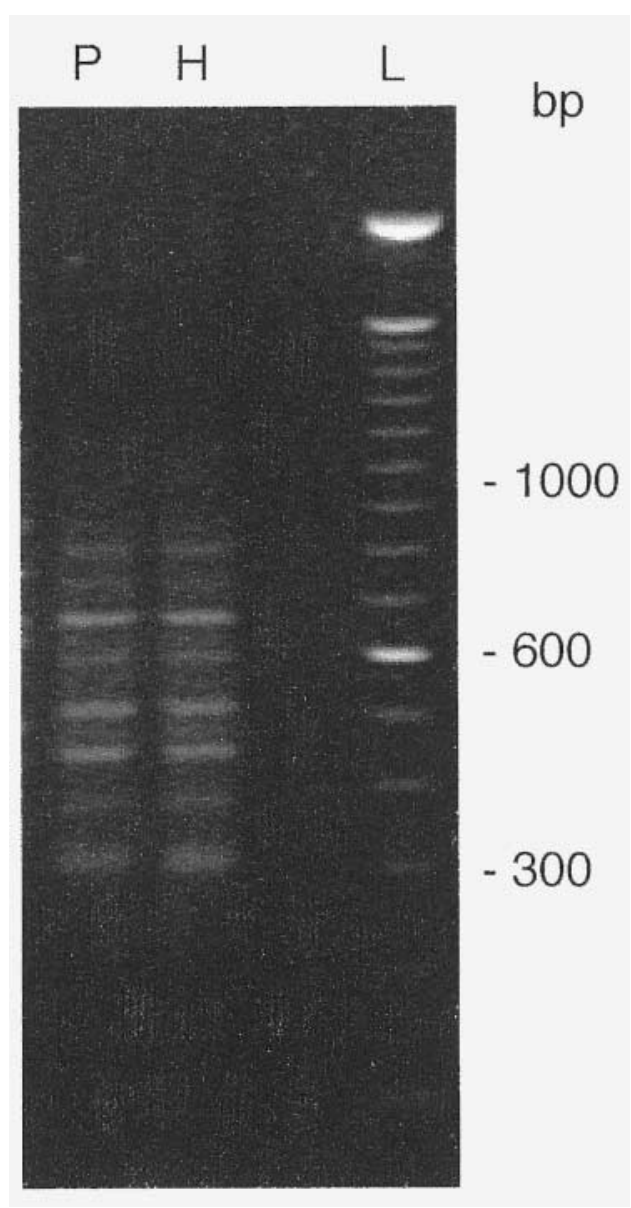

Fig. 1. B. cereus amplified fragment length polymorphism (AFLP) binding patterns from patient isolate (P) and heroin (H) [16]; L, 100-bp ladder. (Figure kindly supplied by Dr J. McLauchlin.) 
antibiogram, i.e., susceptible to erythromycin, gentamicin and vancomycin and resistant to penicillin, amoxicillin and cephalosporins. The heroin isolate was similarly stored at $-70^{\circ} \mathrm{C}$.

\section{Molecular and serological typing}

$B$. cereus isolates were referred for confirmation and typing to the PHLS Food Safety Microbiology Laboratory, Central Public Health Laboratory, Colindale, London. Both isolates were confirmed as B. cereus as described previously [13]; were non-serotypable [14, 15], and showed an indistinguishable amplified fragment length polymorphism (AFLP) profile (Fig. 1) [16].

Genotyping was performed on two selected genes, $g l p \mathrm{~T}$ and $A n s \mathrm{~B}[17,18]$. The differences were $1 \mathrm{bp}$ for $g l p \mathrm{~T}$ and $1 \mathrm{bp}$ for $A n s \mathrm{~B}$ (Fig. 2). These sequencing results showed that the two strains are highly similar but not so closely related to $B$. anthracis as some of the other B. cereus strains included.

\section{Discussion}

The clinical appearance of patients with clostridial gas gangrene is attributed to the production of toxins; it is assumed, although less well investigated, that $B$. cereus also produces toxins that are responsible for clinical signs and symptoms [19]. Its toxin library is known to include the emetic and diarrhoeal toxins more often associated with food poisoning [20]. Both the diarrhoeal toxin and a haemolysin cause dermonecrotic reactions in rabbits [20]. B. cereus is remarkably similar to the better-known and certainly more virulent B. anthracis $[18,20]$.

The patient described in this report was undoubtedly severely ill at presentation, although the presumed focus of his infection appeared unremarkable. Toxic effects often manifest themselves relatively early in the infection process, with little to see both clinically and at operation. Only minimal bacterial growth is required to allow the secretion of potent toxins that may be responsible for significant clinical signs. Such infections progress so rapidly that clinical attention comes too late to save the patient [21]. Early surgical intervention, i.e., incision and debridement of necrotic tissue, is vital in the management of these patients [22]. An additional complication is that the antibiotics of choice for gas-forming bacterial infections are penicillin and metronidazole, as Clostridium spp. are the more usual aetiological agents of these conditions. $B$. cereus is inherently resistant to penicillin, cephalosporins and metronidazole; a patient with crepitant cellulitis or gas gangrene might not receive antibiotics other than $\beta$-lactam agents and metronidazole and these would not be effective against $B$. cereus.

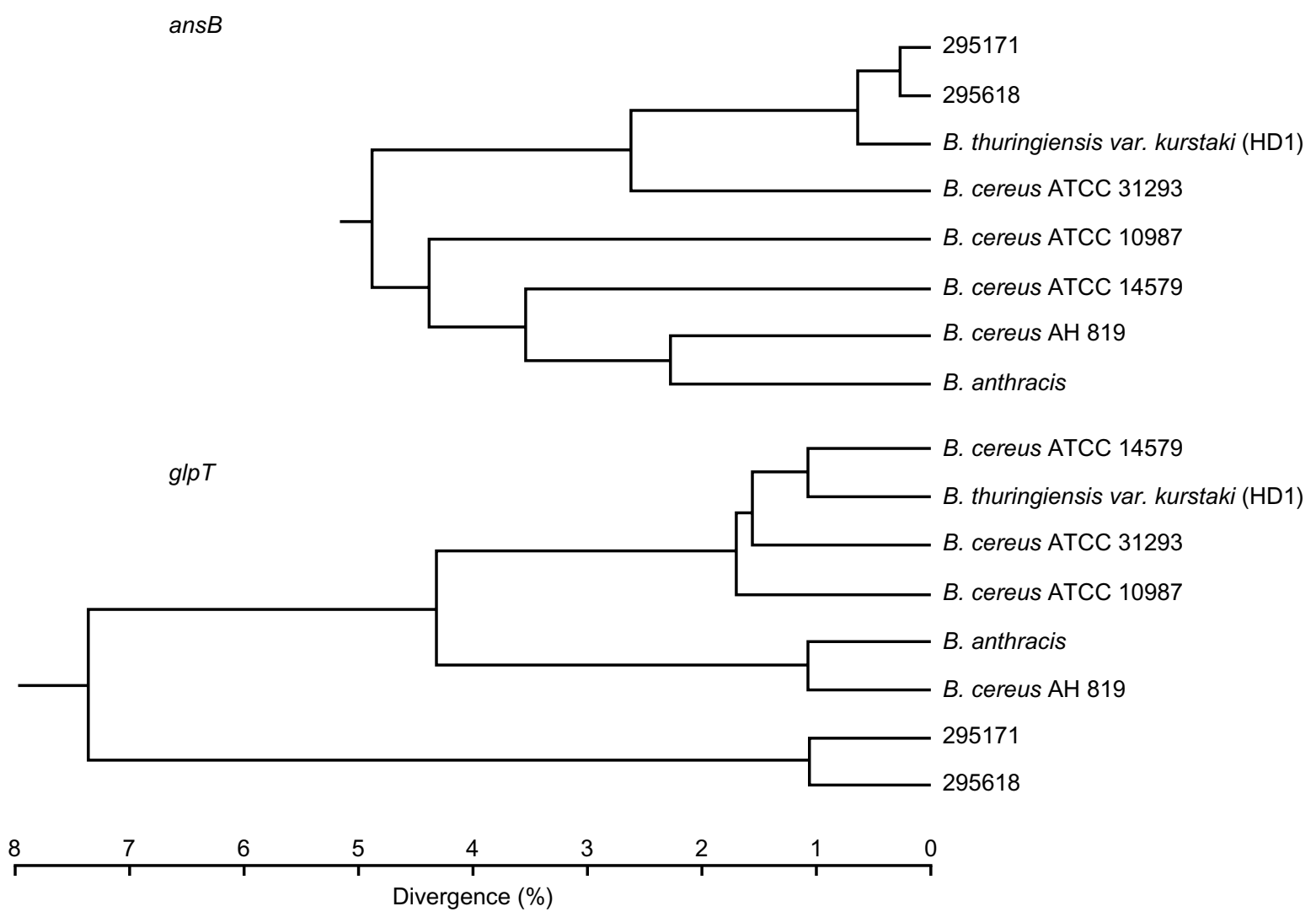

Fig. 2. Dendrograms of two genes found in B. cereus: $g l p \mathrm{~T}$ and $A n s \mathrm{~B}$. Percent divergence is measured from the scale provided. $B$. cereus isolate from the patient is annotated 295171, and the isolate from heroin, 295618. They show $<1 \%$ divergence from each other in both genes. 
Gas in the tissues more commonly suggests anaerobic organisms, but many members of the genus Bacillus are facultative anaerobes [20]. In the present study the isolates actually grew better under anaerobic conditions. There was aerobic growth, but it appeared more slowly and was less profuse, which does not reflect the usual properties of $B$. cereus.

The markedly abnormal liver enzymes seen on admission were initially thought to represent chronic infection with hepatitis $\mathrm{C}$ virus. However, the results of tests performed 6 months previously were essentially normal, and those taken 2 weeks after admission had also returned to normal. It is possible that sepsis from any cause worsens the hepatic enzyme profile with or without hepatitis $\mathrm{C}$, or specific infection with $B$. cereus may exaggerate abnormal liver function, due to systemic effects from the toxin(s) [23]. Fatal liver failure has been described following ingestion of $B$. cereus-contaminated pasta [24].

AFLP typing strategies suggested that the two strains were indistinguishable (Fig. 1). The chance of identical AFLP patterns between unrelated isolates from the patient and his heroin is extremely unlikely. Genotyping confirmed that the strains were more or less identical (Fig. 2).

In conclusion, this report describes a case of $B$. cereus cellulitis in an injecting drug user, which mimicked clostridial sepsis, and was almost certainly caused by the presence of the organism in contaminated heroin. Molecular typing strategies demonstrated a conclusive microbiological link between the patient and his injected heroin, possibly the first report to do so for $B$. cereus. This pathogen should never be underestimated, particularly in relation to its natural resistance to widely used antibiotics.

We thank Dr Jim McLauchlin at the PHLS Food Safety Microbiology Laboratory, Central Public Health Laboratory, Colindale, London, for flagellar and AFLP typing. We also acknowledge Drs Peter Turnbull and Niall Logan for advice and critical review of the text, Erlendur Helgason for preparing the dendrogram, Mrs Su Byrne for secretarial services and all members of the Microbiology Laboratory at the Vale of Leven District General Hospital.

\section{References}

1. Graham CA, McNaughton GW, Crawford R. 'Popping': a cause of soft tissue sepsis in chronic drug abusers. Eur $J$ Emerg Med 1999; 6: 259-261.

2. Hughes RA. Drug injectors and the cleaning of needles and syringes. Eur Addict Res 2000; 6: 20-30.
3. Hinchliffe CE, Thornton GF. Bacillus cereus bacteremia in an intravenous drug abuser. Conn Med 1987; 51: 362-365.

4. Weller PF, Nicholson A, Braslow N. The spectrum of Bacillus bacteremias in heroin addicts. Arch Intern Med 1979; 139. 293-294.

5. Johnson DA, Aulicino PL, Newby JG. Bacillus cereus-induced myonecrosis. J Trauma 1984; 24: 267-270.

6. Meredith FT, Fowler VG, Gautier M, Corey GR, Reller LB. Bacillus cereus necrotizing cellulitis mimicking clostridial myonecrosis: case report and review of the literature. Scand $J$ Infect Dis 1997; 29: 528-529.

7. al-Hemidan A, Byrne-Rhodes KA, Tabbara KF. Bacillus cereus panophthalmitis associated with intraocular gas bubble. $\mathrm{Br} J$ Ophthalmol 1989; 73: 25-28.

8. Fitzpatrick DJ, Turnbull PCB, Keane CT, English LF. Two gasgangrene-like infections due to Bacillus cereus. Br J Surg 1979; 66: 577-579.

9. Moustoukas NM, Nichols RL, Smith JW, Garey RE, Egan RR. Contaminated street heroin. Relationship to clinical infections. Arch Surg 1983; 118: 746-749.

10. SCIEH. Serious unexplained illness among drug injectors in Scotland: update. SCIEH Weekly Report 2000; 34: 121.

11. Holbrook R, Anderson JM. An improved selective and diagnostic medium for the isolation and enumeration of Bacillus cereus in foods. Can J Microbiol 1980; 26: 753-759.

12. Logan NA, Berkeley RCW. Identification of Bacillus strains using the API system. J Gen Microbiol 1984; 130: 1871-1882.

13. Parry JM, Turnbull PCB, Gibson JR. A colour atlas of Bacillus species. Ipswich, Wolf Medical. 1983.

14. Taylor AJ, Gilbert RJ. Bacillus cereus food poisoning: a provisional serotyping scheme. J Med Microbiol 1975; 8: $543-550$.

15. Gilbert JM, Parry JM. Serotypes of Bacillus cereus from outbreaks of food poisoning and from routine foods. $J \mathrm{Hyg}$ 1977; 78: 69-74

16. Ripabelli G, McLauchlin J, Mithani V, Threfall EJ. Epidemiological typing of Bacillus cereus by amplified fragment length polymorphism. Lett Appl Microbiol 2000; 30: 358-363.

17. Helgason E, Caugant DA, Olsen I, Kolstø A-B. Genetic structure of population of Bacillus cereus and B. thuringiensis isolates associated with periodontitis and other human infections. J Clin Microbiol 2000; 38: 1615-1622.

18. Helgason E, Okstad OA, Caugant DA et al. Bacillus anthracis, Bacillus cereus, and Bacillus thuringiensis - one species on the basis of genetic evidence. Appl Environ Microbiol 2000; 66: 2627-2630.

19. Beecher DJ, Olsen TW, Somers EB, Wong ACL. Evidence for contribution of tripartite hemolysin BL, phosphatidylcholinepreferring phospholipase $\mathrm{C}$ and collagenase to virulence of Bacillus cereus endophthalmitis. Infect Immun 2000; 68 : 5269-5276.

20. Logan NA, Turnbull PCB. Bacillus and recently derived genera. In: Murray PR, Baron EJ, Pfaller MA, Tenover FC, Yolken RH (eds) Manual of clinical microbiology, 7th edn. Washington, DC, American Society for Microbiology. 1999: 357-369.

21. Haiart DC, Andrade B, Murie JA. Gas gangrene following intra-arterial injection of oral medication in a drug abuser. Eur J Vasc Surg 1992; 6: 565-566.

22. Gonzalez MH. Necrotising fasciitis and gangrene of the upper extremity. Hand Clin 1998; 14: 635-645.

23. Yokoyama $\mathrm{K}$, Ito $\mathrm{M}$, Agata $\mathrm{N}$ et al. Pathological effect of synthetic cereulide, an emetic toxin of Bacillus cereus, is reversible in mice. FEMS Immunol Med Microbiol 1999; 24: 115-120.

24. Mahler H, Pasi A, Kramer JM et al. Fulminant liver failure in association with the emetic toxin of Bacillus cereus. $N$ Engl $J$ Med 1997; 336: 1142-1148. 